\title{
A FEW REMARKS ON AN EMBEDDING INTO THE SET OF MEASURES
}

\author{
Piotr Puchała \\ Department of Mathematics, Czestochowa University of Technology \\ Częstochowa, Poland \\ piotr.puchala@im.pcz.pl,p.st.puchala@gmail.com
}

Received: 9 September 2021; Accepted: 7 November 2021

\begin{abstract}
We continue considerations concerning Young measures associated with bounded measurable functions from a recent article. We look at them as at the weak* measurable, measure-valued mappings. We show examples explaining that we cannot regard a Young measure (i.e. a weak ${ }^{*}$-measurable mapping) $\delta_{u(x)}$ as an explicit form of a Young measure associated with a function $u$. We also consider convergence of the sequences of Young measures.
\end{abstract}

MSC 2010: 46N10, 74B99

Keywords: Young measures, optimization, engineering

\section{Introduction}

We begin with recalling the basic ideas and the notation concerning Young measures. In the article [1] the basic ideas, engineering motivations and bibliographic suggestions are presented with more detail.

The ideas leading to the discovery of Young measures lie in the calculus of variations. We minimize the integral functional

$$
\mathscr{J}: V \ni u \rightarrow \mathscr{J}(u):=\int_{\Omega} f(x, u(x), \nabla u(x)) d x \in \mathbb{R} .
$$

In the above formula:

- the set $\Omega$ is a nonempty, bounded open subset of the space $\mathbb{R}^{d}$ with sufficiently smooth boundary $\partial \Omega$ and having positive Lebesgue measure $M$;

- the function

$$
u: \Omega \rightarrow \mathbb{R}^{l}
$$

belongs to a suitable, depending on a particular problem, normed space $V$ (it is usually a Sobolev one); 
- the function

$$
f: \Omega \times \mathbb{R}^{d} \times \mathbb{R}^{l \times d} \rightarrow \mathbb{R}
$$

satisfies the Carathéodory conditions, namely:

- the function

$$
\Omega \ni x \mapsto f(x, s, \xi) \in \mathbb{R}
$$

is measurable for all $(s, \xi) \in \mathbb{R}^{d} \times \mathbb{R}^{l \times d}$;

- for almost all, with respect to the Lebesgue measure on $\Omega, x \in \Omega$ the function

$$
\mathbb{R}^{d} \times \mathbb{R}^{l \times d} \ni(s, \xi) \mapsto f(x, s, \xi) \in \mathbb{R}
$$

is continuous.

Additionally, we want $\mathscr{J}$ to be bounded from below and coercive. The latter means that

$$
\lim _{\|u\| \rightarrow \infty} \mathscr{J}(u) \rightarrow \infty
$$

where $\|\cdot\|$ is a norm on $V$. In this case all the minimizing sequences of $\mathscr{J}$ are bounded, so by the Banach-Alaoglu theorem they have weakly (or weakly*) convergent subsequences. (Further on, the phrase weakly or weakly* will be written as weakly $\left(^{*}\right)$ ).

Remark 1 The special case $d=l=3$ of (1) has a physical interpretation. Namely, the function $u$ is the displacement of the elastic body $\Omega$ having the density $f$ of the internal energy. The $3 \times 3$ matrix $\nabla u(x)$ is called the deformation matrix and can be regarded as a measure of a local strain. The functional $\mathscr{J}$ is called the energy functional. Functionals of this form are often considered in engineering, in particular in elasticity theory.

It is known that if there exist a summable function $k: \Omega \rightarrow \mathbb{R} \cup\{0\}$ and a number $C \geq 0$ such that the integrand $f$ satisfies the inequalities

$$
0 \leq f(x, s, \xi) \leq k(x)+c\left(|s|^{2}+|\xi|^{2}\right)
$$

then the lower semicontinuity in the weak topology of a Sobolev space $H^{1}\left(\Omega ; \mathbb{R}^{l}\right)$ of the functional $\mathscr{J}$ is equivalent to the quasiconvexity of the function

$$
\mathbb{R}^{l \times d} \ni \xi \mapsto f(x, s, \xi) \in \mathbb{R} .
$$

In this case the direct methods of the calculus of variations are applicable, see for example [2] or [3]. 
The problem is more complicated when the integrand $f$ is not quasiconvex, because in this case the functional $\mathscr{J}$, although bounded from below, does not attain its infimum. The minimizing sequences are divergent in the strong topology, but they are convergent weakly $\left({ }^{*}\right)$. Their elements are functions oscillating rapidly around the limit. Moreover, in general, for the weakly $\left(^{*}\right)$ convergent to $u_{0}$ minimizing (sub)sequence $\left(u_{n}\right)$ we have

$$
w\left(^{*}\right)-\lim _{n \rightarrow \infty} f\left(x, u_{n}(x), \nabla u(x)\right) \neq f\left(x, u_{0}(x), \nabla u_{0}(x)\right) .
$$

In 1937, in the article [4], Laurence Chisholm Young introduced (in a one dimensional special case) generalized solutions to problems of minimizing integral functionals with integrands such that the regarded functionals do not attain their infima. These generalized solutions are called Young measures. General treatment of Young measures can be found for example in [5-8].

Remark 2 The energy functionals not attaining their infima are also met in engineering. Such are for instance the energy functionals of shape memory materials. Applications of Young measures in engineering can be found for instance in $[3,9,10]$ and the references cited there.

In this article we continue the considerations concerning the existence theorem for Young measures presented in [1]. Our approach is the one described with details in [8]. We refer the reader there for a detailed presentation or to [1] for notational details and necessary results without proofs. Various approaches to Young measures are described in [5]. The basic result for us is the Theorem 3.1.6 from [8].

Theorem 1 (Theorem 3.1.6 in [8]) The diagram is commutative.

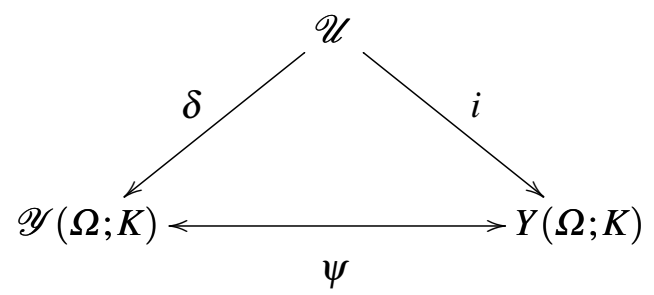

In the above theorem:

- $\mathscr{U}$ is a set of all Borel measurable functions on $\Omega$ with values in a compact set $K \subset \mathbb{R}^{l}$;

- $Y(\Omega, K)$ is the weak* closure of the image $i(\mathscr{U})$ of the set $\mathscr{U}$ under a suitably defined mapping $i: \mathscr{U} \rightarrow L^{1}(\Omega, C(K))^{*}$;

- $\mathscr{Y}(\Omega, K) \subset L_{w^{*}}^{\infty}(\Omega, \operatorname{rca}(K))$ is a set of weakly* measurable functions from the set $\Omega$ into the set of regular, countably additive (rca) probability measures on $K$. The values of weakly* measurable functions from $\mathscr{Y}$ are the Young measures on the set $K$; 
- a mapping $\psi: L_{w^{*}}^{\infty}(\Omega, \operatorname{rca}(K)) \rightarrow L^{1}(\Omega, C(K))^{*}$ is an isometric isomorphism;

- a Dirac mapping $\delta$ is defined by $\delta(u)(x):=\delta_{u(x)}$, where $\delta_{k}$ is a Dirac measure at the point $k$. The mapping $\delta$ is an embedding of the set $\mathscr{U}$ of functions into the set of Young measures defined on the range of these functions.

Remark 3 In [8], the references for generalizations of the basic results concerning Young measures to an unbounded set $\Omega$ or the measures on $\Omega$ that are more general than the Lebesgue one, are given.

\section{An embedding of functions into the set of Young measures}

From a practical point of view, the most important problem is obtaining a method of finding an explicit form of the Young measures in specific cases. The reader is referred to [11] and [12] for such a method in case of oscillating functions. However, the problem of at least equal importance is the general characterization of functions for which there exist the associated Young measures. The Theorem 1 provides an answer, because on the basis of this theorem we can infer the existence of a Young measure associated with any bounded Borel fuction. This makes futher characterizations possible, like the probabilistic one for example, which additionally enables employing computer methods to simulation Young measures associated with bounded measurable functions, see [13].

Having in mind the already known assumptions, we know that if $\left(u_{n}\right)$ is a sequence of bounded Borel functions, $\varphi$ - a continuous real function on $\mathbb{R}^{l}$, then the weak $\left({ }^{*}\right)$ limit of the (sub)sequence $\left(\varphi\left(u_{n}\right)\right)$ can be expressed as

$$
\int_{\Omega} \bar{\varphi}(x) w(x) d x
$$

Here, $w$ is any integrable real function, while $\bar{\varphi}$ is the function of the form

$$
\bar{\varphi}(x)=\int_{K} \varphi(s) v_{x}(d s) .
$$

The family $\left(v_{x}\right)_{x \in \Omega}$ of probability measures on $K$ is called the Young measure associated with the sequence $\left(u_{n}\right)$ or parametrized measure associated with the sequence $\left(u_{n}\right)$. Observe that in general, $\left(v_{x}\right)_{x \in \Omega}$ is a range of a weakly* measurable mapping

$$
v: \Omega \ni x \rightarrow v(x) \in \operatorname{rca}^{1}(K),
$$

where elements of the set $\operatorname{rca}^{1}(K) \subset \operatorname{rca}(K)$ are probability measures. Thus $v \in \mathscr{Y}(\Omega, K)$. If the mapping $v$ is constant, we call the related Young measure homogeneous one. This in particular means that a family $\left(v_{x}\right)_{x \in \Omega}$ of probability measures consists of one element only (which of course means that it does not depend on $x \in \Omega$ ). A simple characterization of homogeneous Young measures can be found 
in [12]. Observe that through the isometric isomorphism $\psi$ in Theorem 1, Young measures associated with functions can be identified as weak ${ }^{*}$ limits of appropriate sequences of compositions.

Consider the Dirac embedding $\delta$ in Theorem 1. The value of $\delta$ on the function $u \in \mathscr{U}$ is $\delta_{u(x)}, x \in \Omega$. In the article [1] a mapping $\varkappa$, from the set $\mathscr{U}$ into the set $\mathscr{Y}(\Omega, K)$ of Young measures, has been introduced as follows. Let $\mu$ be a Lebesgue measure on $\Omega$ and $M:=\mu(\Omega)$. Then for $u \in \mathscr{U}, A \in \mathscr{B}(K)$ let

$$
\Omega \ni x \rightarrow(\varkappa(u))(x):=\frac{m_{A}}{M} \delta_{u(x)}(\cdot)
$$

where

$$
m_{A}:= \begin{cases}\mu\left(u^{-1}(A)\right), & \text { if } u(x) \in A \\ 0, & \text { if } u(x) \notin A .\end{cases}
$$

(We of course have $m_{K}=M$.) It has been also proved there, that if $v^{u}$ is a Young measure associated with $u$, then $v^{u}(A)=\mu\left(u^{-1}(A)\right) \delta_{u(x)}(A)$ for any Borel subset $A$ of $K$.

The motivation for $\varkappa$ is that we should not look at the values of $\delta$ as the explicit form of the Young measures associated with functions from $\mathscr{U}$. This is illustrated by the following examples.

Example 1 Consider a function $u:(0,6) \rightarrow \mathbb{R}$ defined by:

$$
u(x):= \begin{cases}2, & x \in(0,2] \\ 5, & x \in(2,5] . \\ 9, & x \in(5,6)\end{cases}
$$

It is known (see [11] for direct proof for simple functions), that the Young measure $v^{u}$ associated with $u$ is a homogeneous one. Its explicit form is given by the formula

$$
v^{u}=\frac{1}{3} \delta_{2}+\frac{1}{2} \delta_{5}+\frac{1}{6} \delta_{9}
$$

If we looked at the values $\delta(u)$ of the Dirac embedding $\delta$ taken at $u \in \mathscr{U}$ as a Young measure, in its explicit form, associated with $u$, then we could consider it as a family $\left\{\delta_{u(x)}\right\}_{x \in(0,6)}$, so we would have

$$
\left\{\delta_{u(x)}\right\}_{x \in(0,6)}=\left\{\delta_{2}, \delta_{5}, \delta_{9}\right\},
$$

which could be written as

$$
\delta(u)(x)=\delta_{u(x)}= \begin{cases}\delta_{2}, & x \in(0,2] \\ \delta_{5}, & x \in(2,5] \\ \delta_{9}, & x \in(5,6)\end{cases}
$$


Let $A:=(1,3)$. Then we have $\delta_{u(x)}(A)=\delta_{2}(A)=1$, while the actual Young measure of this set is equal to

$$
v^{u}(A)=\frac{1}{3}=\varkappa(u)(x)(A)=\frac{\mu\left(u^{-1}(A)\right)}{M} \delta_{u(x)}(A) .
$$

Now let $B:=(1,3) \cup(4,7)$. Then for the Dirac embedding we would have

$$
\delta_{u(x)}(B)=\delta_{2}((1,3))+\delta_{5}((4,7))=2,
$$

which is obviously not the Young measure of $B$. The latter is actually equal to

$$
v^{u}(B)=\frac{1}{3}+\frac{1}{2}=\frac{5}{6}=\varkappa(u)(x)(B)=\frac{\mu\left(u^{-1}(B)\right)}{M} \delta_{u(x)}(B) .
$$

Now consider a case of a Young measure with density.

Example 2 Let $u(x):=2 x, x \in(0,2)$. Then (see [11] for direct proof for monotonic functions) the Young measure associated with $u$ is a homogeneous one and is absolutely continuous with respect to the Lebesgue measure $d y$ on the set $[0,4]$, with density being the constant function equal to $\frac{1}{4}$ on this interval. That is, $v^{u}=\frac{1}{4} d y$. The set $\left\{\delta_{2 x}\right\}_{x \in(0,2)}$ is an uncountable set of Dirac measures.

Let $A:=\{1\}$. Then $\delta_{u(x)}(A)=1$, while $v^{u}(A)=0$, because the Lebesgue measure of any countable set is zero. Since $u^{-1}(A)=\left\{\frac{1}{2}\right\}$ then

$$
\varkappa^{u}(A)=\mu\left(\left\{\frac{1}{2}\right\}\right) \delta_{u(x)}(\{1\})=0 \cdot 1=0 .
$$

Let us take into consideration a Carathéodory integrand $h$. It is an element of the space $\operatorname{Car}(\Omega, K ; \mathbb{R})$ of real functions defined on the product $\Omega \times K$. Elements of this space satisfy the Carathéodory conditions. This means that for any $h \in \operatorname{Car}(\Omega, K ; \mathbb{R})$, the function $h(\cdot, k), k \in K$, is measurable, while the function $h(x, \cdot), x \in \Omega$, is continuous. We equip this space with a norm given by the formula: for all $h \in \operatorname{Car}(\Omega, K ; \mathbb{R})$

$$
\|h\|_{\text {Car }}:=\int_{\Omega} \sup _{k \in K}|h(x, k)| d x .
$$

Let us denote by $L^{1}(\Omega, C(K))$ the space of those functions denoted on $\Omega \subset \mathbb{R}^{d}$ with values in a vector space $C(K)$, which are integrable in a Bochner sense. It can be proved that this space is isometrically isomorphic to the space $\operatorname{Car}(\Omega, K ; \mathbb{R})$, so these spaces can be identified.

Observe, that for any $h \in L^{1}(\Omega, C(K)$ there holds

$$
\begin{gathered}
\int_{\Omega} \int_{K} h(x, k)(\varkappa(u)(x))(d k) d x=\int_{\Omega} \int_{K} h(x, k) \frac{\mu\left(u^{-1}(K)\right)}{M}\left(\delta_{u(x)}\right)(d k) d x= \\
=\int_{\Omega} \int_{K} h(x, k) \delta_{u(x)}(d k) d x=\int_{\Omega} h(x, u(x)) d x,
\end{gathered}
$$


so the first equation in the proof of the Theorem 1 remains true with $\delta$ replaced by $\varkappa$. Thus, from the Theorem 2 in [1] and the relation (3), it follows that we can derive the following conclusion from the Theorem 1.

Corollary 1 For any Borel function $u: \Omega \rightarrow K$ there exists a Young measure $v^{u}$ associated with $u$. It is a regular probability measure on $K$ such that if $A \in \mathscr{B}(K)$ then $v^{u}(A)=\frac{m_{A}}{M} \delta_{u(x)}(A)$.

Recall that a sequence $\left(v_{n}\right)$ of bounded measures on a compact set $K \subset \mathbb{R}^{l}$ converges weakly* to a measure $v_{0}$, if $\forall \beta \in C(K, \mathbb{R})$ there holds

$$
\lim _{n \rightarrow \infty} \int_{K} \beta(k) d v_{n}(k)=\int_{K} \beta(k) d v_{0}(k) .
$$

Let $u \in \mathscr{U}$ and let $\left(u_{n}\right)$ be a sequence of simple functions from $\mathscr{U}$ converging uniformly to $u$. Such a sequence always exists since the elements of $\mathscr{U}$ are bounded functions. Moreover, the element $u_{n}, n \in \mathbb{N}$, can be constructed with respect to a suitably constructed finite partition $\bigcup_{i=1}^{l(n)} K_{i}^{(n)}$ (of arbitrarily small diameter) of the set $K$, see e.g. [14]. Therefore, in particular $u_{n}$ takes the values $p_{i}^{(n)}, i=1,2, \ldots, l(n)$.

Let $\left(v_{n}\right)$ be a sequence of the Young measures associated with the respective ele-

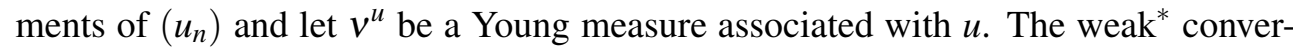
gence of the sequence $\left(v_{n}\right)$ to $v^{u}$ follows from the calculations

$$
\begin{gathered}
\left|\int_{K} \beta(k) v_{n}(d k)-\int_{K} \beta(k) v^{u}(d k)\right|=\left|\int_{K} \beta(k) \varkappa_{n}(d k)-\int_{K} \beta(k) \varkappa^{u}(d k)\right|= \\
=\frac{1}{M}\left|\sum_{i=1}^{l(n)} m_{K_{i}^{(n)}}^{n} \int_{K_{i}^{(n)}} \beta(k) \delta_{u_{n}(x)}(d k)-\sum_{i=1}^{l(n)} m_{K_{i}^{(n)}} \beta K_{K_{i}^{(n)}} \beta(k) \delta_{u(x)}(d k)\right| \leq \\
\leq \frac{1}{M} \sum_{i=1}^{l(n)}\left|m_{K_{i}^{(n)}}^{n} \beta\left(p_{i}^{(n)}\right) \delta_{u(x)}\left(K_{i}^{(n)}\right)-m_{K_{i}^{(n)}} \beta(u(x)) \delta_{u(x)}\left(K_{i}^{(n)}\right)\right|= \\
=\frac{1}{M} \sum_{i=1}^{l(n)}\left|m_{K_{i}^{(n)}} \beta(u(x)) \delta_{u(x)}\left(K_{i}^{(n)}\right)-m_{K_{i}^{n}}^{n} \beta\left(p_{i}^{(n)}\right) \delta_{u(x)}\left(K_{i}^{(n)}\right)\right| \leq \\
\leq \sup _{u(x) \in K}|\beta(u(x))| \frac{1}{M} \sum_{i=1}^{l(n)}\left|m_{K_{i}^{(n)}}-m_{K_{i}^{(n)}}^{n}\right|+ \\
+\frac{1}{M} \sum_{i=1}^{l(n)} m_{K_{i}^{(n)}}^{n}\left|\beta(u(x)) \delta_{u(x)}\left(K_{i}^{(n)}\right)-\beta\left(p_{i}^{(n)}\right) \delta_{u(x)}\left(K_{i}^{(n)}\right)\right| .
\end{gathered}
$$

We have observed above that Young measures are regular, countably additive probability measures on the set $K$. The set of all regular, countably additive measures on $K$ is denoted by $\operatorname{rca}(\mathrm{K})$. When equipped with the total variation norm, 
this set becomes a Banach space (see, for example, paragraph 1.4 of [8] and the references there). We can thus consider weak convergence in $\mathrm{rca}(\mathrm{K})$ with this norm. The set of Young measures on $K$ is a subset of the unit sphere of this Banach space. We will use the Dieudonné-Grothendieck criterion for weak convergence of measures. See Theorem 6.4.2 in [15].

Let $u \in \mathscr{U}$ and let $\left(u_{n}\right)$ be a sequence of functions from $\mathscr{U}$ converging uniformly to $u$. Let $A \in \mathscr{B}(K)$ be any fixed open set. Then for any fixed $x \in \Omega$ we have

$$
\begin{gathered}
\left|v_{n}(A)-v^{u}(A)\right|=\left|\int_{A} v_{n}(d k)-\int_{A} v^{u}(d k)\right|= \\
=\frac{1}{M}\left|\int_{A} \mu\left(u_{n}^{-1}(A)\right) \delta_{u_{n}(x)}(d k)-\int_{A} \mu\left(u^{-1}(A)\right) \delta_{u(x)}(d k)\right|= \\
=\frac{1}{M}\left|\mu\left(u_{n}^{-1}(A)\right) \delta_{u_{n}(x)}(A)-\mu\left(u^{-1}(A)\right) \delta_{u(x)}(A)\right| .
\end{gathered}
$$

Let $u(x) \in A$. Due to the uniform convergence of $\left(u_{n}\right)$ to $u$, the expression

$$
\mu\left(u_{n}^{-1}(A)\right) \delta_{u_{n}(x)}(A)-\mu\left(u^{-1}(A)\right) \delta_{u(x)}(A)
$$

vanishes for all $n$ greater than certain $n_{0}$. The same takes place if $u(x)$ is not an element of the closure of $A$. If $u(x)$ belongs to the boundary of $A$ then $\delta_{u(x)}(A)=0$. Moreover, for all $n$ large enough there holds the inequality $\mu\left(u_{n}^{-1}(A)\right) \leq \mu\left(u^{-1}(B(u(x), \varepsilon)), \varepsilon>0\right.$. Thus $\mu\left(u_{n}^{-1}(A)\right) \delta_{u_{n}(x)}(A) \rightarrow 0$ as $n \rightarrow \infty$. Since the above considerations are valid for all open sets in $K$, by the Dieudonné Grothendieck criterion the Young measure $v^{u}$ is the weak limit of the sequence $\left(v_{n}\right)$.

\section{Conclusions}

From the point of view of applied sciences, one of the most important issues concerning Young measures is the existence of method(s) allowing for the calculation of their explicit form or measures of specific sets. The Dirac embedding $\delta$ is used in proving the fundamental fact of the existence of Young measures associated with functions. However, its values at particular points (that is, at the functions from $\mathscr{U}$ ) can not be looked at as the Young measures, in their explicit form, associated with these functions, as it has been shown in the simple examples. The embedding $\varkappa$, as it is shown in [1], 'hits' the Young measure associated with particular function $u \in \mathscr{U}$ directly. This, together with the Corollary 1, makes the existence theorem more intuitive. Obviously, the values of $\varkappa$ also do not provide explicit forms of Young measures, but allow for the calculation of the Young measures of particular sets, which is not the case when considering $\delta$. It also links the Young measure associated with the function $u$ with the domain of definition of this function. The convergence results show that $v^{u}(A), u \in \mathscr{U}, A \in \mathscr{B}(K)$, can be approximated by $v_{n}(A)$, where $v_{n}$ is a Young measure associated with an appropriate simple function. Since an explicit 
form of a Young measure associated with a simple function is well known, this makes the solving of such problems much easier.

\section{References}

[1] Puchała, P. (2021). On a certain embedding in the space of measures. J. Appl. Math. Comput. Mech., 20(2), 53-63.

[2] Dacorogna, B. (2008). Direct Methods in the Calculus of Variations. Springer Science+Business Media, LLC.

[3] Kružík, M., \& Roubíček, T. (2019). Mathematical Methods in Continuum Mechanics of Solids. Springer Nature.

[4] Young, L.C. (1937). Generalized curves and the existence of an attained absolute minimum in the calculus of variations. Comptes Rendus de la Société des Sciences et des Lettres de Varsovie, classe III, 30, 212-234.

[5] Florescu, L.C., \& Godet-Thobie, Ch. (2012). Young Measures and Compactness in Measure Spaces, Walter de Gruyter GmbH \& Co. KG.

[6] Pedregal, P. (1997). Parametrized Measures and Variational Principles. Birkhäuser.

[7] Rindler, P. (2018). Calculus of Variations. Springer International Publishing AG, Part of Springer Nature.

[8] Roubíček, T. (1997). Relaxation in Optimization Theory and Variational Calculus. Walter de Gruyter.

[9] Müller, S. (1999). Variational Models for Microstructure and Phase Transitions. Calculus of variations and geometric evolution problems. Lecture Notes in Mathematics, (1713), Springer, 85-210.

[10] Pedregal, P. (2000). Variational Methods in Nonlinear Elasticity. Society for Industrial and Applied Mathematics.

[11] Puchała, P. (2014). An elementary method of calculating Young measures in some special cases. Optimization, 63(9), 1419-1430.

[12] Puchała, P. (2017). A simple characterization of homogeneous Young measures and weak $L^{1}$ convergence of their densities. Optimization, 66(2), 197-203.

[13] Grzybowski, A.Z., \& Puchała, P. (2019). Classical Young Measures Generated by Oscillating Sequences with Uniform Representation. Transactions on Engineering Technologies. Springer Nature Singapore Ptu Ltd., 1-12.

[14] Aliprantis, Ch.D., \& Border, K.C. (1999). Infinite Dimensional Analysis. A Hitchhiker's Guide. Berlin-Heidelberg: Springer-Verlag.

[15] Benedetto, J.J., \& Czaja, W. (2009). Integration and Modern Analysis. Boston: Birkhäuser. 